\title{
The next generation in the evolution of global surgery
}

\author{
Janet Chao ${ }^{1}$ and Robert Pearl ${ }^{2 *}$ \\ ${ }^{1}$ Medical student, George Washington School of Medicine, USA, janetchao@gwmail.gwu.edu \\ ${ }^{2}$ Department of Plastic Surgery, Stanford School of Medicine, USA, Robert.Pearl@kp.org
}

For decades, surgeons from the United States have volunteered to travel to areas of the world with shortages of physicians to provide surgical care to patients who otherwise would not have access. Teams of physicians, nurses, and support personnel spend one to two weeks in a given location, working with local community leaders, doctors and hospitals to provide clinical expertise.

Depending on the specific composition of the team, surgical care may include cataract surgery, repair of cleft lip and palate, hand reconstruction, and burn deformity repair, to name just a few of the most commonly performed procedures.

These visiting surgical teams have helped tens of thousands of individuals to have an improved life and allowed them to earn a living, raise their family, and become strong contributors to their community. Since these surgical teams began volunteering around the world, for such missions, global health care has evolved; still, many challenges exist. To address these challenges and advance global surgery in important and meaningful ways, we believe that the next generation of global surgical care must continue to adapt to the changing environment.

The rise of globalization and an increased need for collaboration will require changes in how care is provided and clinicians are trained. This next generation in global health, which we call "Generation 3," will leverage advanced 21 st century technology and improve surgical care by shifting the expertise provided from intermittent to continual.

Unlike Generation 1, where much of the surgical care was performed by the U.S. resident, or even Generation 2 in which local surgeons are trained once or twice a year by visiting American surgeons, Generation 3 will be relationship based and encourage continuous, long-term collaboration between the volunteer organization and the destination community.

This next generation model has the potential to create integrated global networks, capable of rapid transfer of information and learning. The recent Ebola, Mers and Zika epidemics have demonstrated the importance of this type of global cooperation for infectious diseases. In a recent article, published in the New England Journal of Medicine, "Partnerships, Not Parachutes, for Zika Research," the authors conclude that, "If open sharing of data and specimens becomes the norm among scientists and epidemiologists around the world, we will be far more likely to succeed in improving international public health capacity and strengthening our collective health and human security" [2]. Global surgery has the potential to be the next frontier in accomplishing this important objective.

\section{Generation one - The medical mission to maximize direct patient care}

The first generation of global volunteerism was designed primarily to provide surgical expertise to patients who otherwise could not obtain it. These trips also were organized to expand the training of the American residents. Success was measured in terms of the number of procedures that could be completed in a short amount of time. Broad participation by U.S. trainees allowed more teams to be sent and more procedures to be completed; however, the model created a variety of difficulties. First, there were conflicts with local surgeons who believed that the American surgeons and their residents were interlopers and complained that these trips took advantage of the local population. Second, patient follow-up was minimal, and those individuals who experienced complications or needed additional procedures had to wait until the subsequent team arrived for their problems to be addressed. In response to these obstacles, many of the sponsoring institutions modified their approach.

\section{Generation two - The medical mission to maximize local expertise}

The second generation of global health shifted from focusing on completing procedures to educating and training local physicians to perform the procedures. This meant less involvement of U.S. residents and required an expanded commitment from the participating attending physicians.

Dr. David Shaye of the Department of Global Health and Social Medicine and the Department of Otolaryngology at Harvard Medical School described this first evolution of global surgical care as a move from "vertical systems" to "horizontal" and "diagonal systems." The vertical system is the traditional model of global volunteerism, a "mission trip" [1]. Although the individual patients whose problems are addressed in the traditional approach benefit, this model for global surgery lacks continuity and sustainability. Once the mission trip ends and the health care team returns to the United States, the local community must wait for the next team to arrive before additional progress at addressing the community's disease burden occurs. To address this bottleneck, modifications were made to the model. Specifically, the composition and focus of the team shifted from care delivery and the training of U.S. residents to the teaching and education of local doctors, nurses, and staff. As the old saying goes, "Give a man a fish, and you feed him for a day. Teach a man to fish, and you feed him for a lifetime."

Analogous changes in medical training were happening in the U.S at

Correspondence to: Robert Pearl, Department of Plastic Surgery, Stanford School of Medicine, USA, E-mail: Robert.Pearl@kp.org

Received: April 28, 2016; Accepted: May 20, 2016; Published: May 24, 2016 
the same time. Accrediting and regulatory organizations expanded the amount of supervision required by attending physicians, and payment, particularly from Medicare, became dependent on appropriate documentation of direct involvement during the operative procedure itself. As in the global environment, these expanded expectations placed added burden on the participating physicians. That said, these changes were recognized as important from an ethical perspective and thus a step in the right direction.

Although this first evolution in global surgery was an improvement over the traditional model, in practice it proved to be less than perfect. Sometimes, having been trained by American surgeons, the local physicians chose to leave their home area, resulting in a "brain drain" [3]. On other occasions, the local surgeons stayed, but shifted their practice from public to private patients and from reconstructive to cosmetic surgery. Even when the local surgeons remained, the patient care remained fragmented, and the training limited. In particular there was minimal follow-up data collected on outcomes and few interactions with the participating attending physicians from one trip to the next. Finally, from a cultural perspective, the American culture and language continued to dominate, and local physicians were expected to adapt to the language and cultural expectations of the visiting surgical teams, rather than there being bidirectional sharing.

\section{Generation three -- The medical mission to maximize longitudinal collaboration}

Once again, there are parallels between the current challenges in the U.S. and the difficulties encountered in global health care. In spite of the advances that have been made, major disparities in the care provided and the clinical outcomes achieved remain today in the U.S. In both settings, language, cultural and socio-economic differences contribute to the problem. In an attempt to bridge the gaps, leading organizations have used modern technology, particularly video, with increasing success [4].

The growing diversity in American health care is mirrored by the pace of globalization. In an article published in the New England Journal of Medicine, "Convergence to Common Purpose in Global Health," the authors describe the major trends that will impact global health care in the future, and the opportunities that exist. These include the economic and health consequences of globalization, the internationalization of medical knowledge, the globalization of the health workforce, and the globalization of medical science. Thirty years ago, medical knowledge traveled slowly, if at all. Today, thanks to the internet, papers are available online months before publication and instantaneously available anywhere in the world [5].

As a result of these changes, many of the major advances in current medical research are being published by researchers from a growing number of countries. The Commission on Health Research for Development recently reported that the number of countries engaged in "essential national health research" has increased substantially since the late 1980s. A notable example is China, which had been a developing country during the initial 1980s report, but now produces the second highest number of articles in the world [5].

Cultural sensitivity,, both in U.S. and globally, will be an essential part of Generation 3. There already is a movement to modify the formal curriculum of medical education to more explicitly include the values of equitable partnership and collaboration in health care decision making and care delivery. In parallel, global surgery programs will need to evolve in ways that demonstrate respect for the local culture and embrace opportunities that create long-term relationships among the visiting programs, the hosting communities and the local physicians. In the Annals of Surgery, Drs. Mutabdzic and Azzie point out that relative to global health elective courses, "it will take more than formal requirements to change the conversation from one regarding a trainee's incredible experiences to one regarding the benefits and the pitfalls for the hosts and their community. Both conversations are important and should be permitted, but what our host communities think and feel, a topic rarely evaluated or expressed in the literature, will be a much more difficult one [6].

"Generation 3" will focus on an equal relationship and partnership between the host and visiting surgical teams. It will demand the sharing of information, a commitment to providing surgery on multiyear basis, a willingness to examine long-term outcomes and a desire to work together to improve the health of the entire community, not simply those who receive surgery. It will leverage 21 st century technology, particular video in new and innovative ways. Over time, this next generation will create an international community willing to address the challenges patients face across the globe.

This trend is already underway. In 2008, donations were made by both the Bill and Melinda Gates Foundation and the Rockefeller Foundation, to the Consortium of Universities for Global Health to interconnect academic, industry, and philanthropic agencies. This consortium arranges educational meetings and publishes a Web site that offers an open access forum for members to share their experiences with broader communities across the globe [7]. While these efforts are laudable and a step in the right direction, much more needs to be done. The "global surgery workforce is in crisis in many low- and middle-income countries (LMICs)" with a critical need for systemically collected, national-level data regarding surgery providers" [8].

The fragmentation of care is even greater when researchers examine the impact of volunteer visiting teams. Physicians from the University of Wisconsin's Division of Plastic and Reconstructive Surgery conducted a nationwide survey on global health curricula in plastic surgery residency programs. The survey data revealed the relatively fragmented, opaque nature of academic global surgery pursuits. They recommended the formation of a multidisciplinary group, composed of academic leaders from low, middle, and high-income countries that could coalesce and freely disseminate information about the activities and strategies used by individual institutions, thereby improving the current state of ambiguity worldwide [9].

As the leaders of global surgery collaborate to put the pieces together, the impact of their work will be magnified. By moving from procedures to relationships, long-term planning and commitment becomes possible. As the emphasis shifts from completing procedures to working with local leaders and doctors to create new solutions, people who share the language and the culture will become more interested in participating. Through technology, frequent video interactions can be scheduled. When long-term commitment is gained via on-going interactions, continuous education and follow-up, cultural understanding and respect will become increasingly bidirectional, and global health networks will progressively replace single site efforts.

Unfortunately, as powerful as this vision is for the future, and as attractive as it will be for many, getting there will be more difficult than people initially assume. It is one thing to want to bridge language and culture, it is another to accomplish it. 


\section{Experience adjusting to a new culture}

To understand the challenges and opportunities inherent in making this transition, it is helpful to look at the experience one of us (JC) had providing health care in another culture, that of South Korea. As part of her participation in the global health track at The George Washington University School of Medicine, she spent 8 -weeks in Korea completing a summer internship, and subsequently returned to Korea the next year to continue to work on many of the projects she had begun. From an educational perspective, her clinical and research experience during the first summer was exceptional, with major opportunities for clinical learning in head and neck surgery and in-depth scientific exploration. From a language and cultural perspective, however, the first year was trying. Although conversationally fluent in Korean, participating in scientific and medical discussions proved difficult compared to informal social settings. Technical and medical terminology is different than day-today conversational language.

Language is only part of the challenge. Figuring out the nuances of the culture proves equally, if not even more, complex. For example, Korean culture contains a multitude of levels of respect based not just on title, but also on age. These culturally unique standings determine a variety of outcomes including those far beyond medical care, like who pays for drinks and dinner during group outings. Use the wrong word or try to pay when it is not appropriate, and colleagues will be greatly insulted. And in Korea, these out-of-clinic interactions set the tone for in-clinic ones. In Korea physicians and researchers spend long hours at work, but are also expected to participate with their colleagues in frequent dinners and late night events. Refusing to do so is disrespectful. There may be something you want to do at night with your family or your non-medical friends, but declining a work invitation is problematic. The experience of JC highlights some of the challenges that exist in adjusting to a new and unfamiliar culture, even when you have a strong language foundation and a moderate familiarity with the culture. When you arrive without these skills and experiences, the level of discomfort can be overwhelming. We can expect that in the process of shifting from a single trip-based approach to a long-term relationship-based one, that some of the participants will experience these types of cultural barriers.

Though overcoming language and cultural challenges is difficult, the rewards of doing so are great. By the end of the first summer, social time that once felt burdensome had produced deep friendships and a strong sense of collegiality and mutual support. Between her first and second trips to Korea, JC maintained communications with research and clinical colleagues via tools like Skype and Kakao Talk. They shared data, worked on analyses, and wrote and published a series of papers. By her second trip to Korea, JC's relationships were already in place and the cultural divide almost completely closed.
This outcome, a longitudinal relationship with ongoing collaboration is an opportunity the next generation of global surgery will need to seize, particularly through modern informational and mobile technology. To do so will require a long-term commitment to this goal and a deep cultural understanding gained through both formal education and on-the-ground experience.

\section{The patient's experience}

A sometimes unexpected and always rewarding lesson from participating in global surgery is being able to experience care delivery in new and different ways. In this case, the experience of being from outside the dominant culture provides insights into how patients often feel. These same cultural and language discomforts that a medical student or visiting surgeon experiences in an unfamiliar culture, often exists for the patients they treat at home. In the future, those who participate in global health work will be leaders in expanding cultural awareness here in the States.

\section{Recommendations for the Future (Table 1)}

Many people across the globe have access to and are comfortable using wifi and cell phones, a trend that is likely to proliferate even further in the future [10]. These 21st century technologies can facilitate and support an on-going relationship between the local physicians and their counterparts in the United States. They can be used to provide patient follow-up, assistance with unresolved surgical problems and continuous education. This is already beginning via virtual interactive presence and augmented reality (VIPAR), an iPad-based tool that has been used to enable collaborative efforts between Vietnamese and American neurosurgeons to take place [11]. Moving from Generation 2 to Generation 3 will be difficult, but the rewards will be worth it. In particular, the opportunity for a long-term relationship with colleagues from across the globe has the potential to attract an expanded number of medical students, residents and attending physicians with language proficiency and cultural expertise. The opportunity to contribute in a variety of ways, other than a twoweek in-person trip, will make on-going participation possible for individuals during their residency training and when they begin their clinical practice. The result of more frequent collaboration will be greater trust, life-long friendships and shared learning. Through increased continuity, an expanded educational focus and the use of modern technology, the opportunity will exist to document and report on clinical outcomes, moving global health into the same realm as the rest of academic medicine. Finally, through the availability of $21^{\text {st }}$ century technology, teams from different organizations, universities and destination sites will be able to link together in a global health network, exchange information and collaborate on research and clinical practice. Already this is beginning to happen through Csurgeries, an internet based, peer reviewed video journal that "aims

Table 1. Opportunities that come out of the "Generation 3" in the Evolution of Global Surgery.

Expanded follow-up and new research opportunities on Surgical Outcomes

Sharing Resources (ex: development of teaching videos to train local speech therapists following cleft surgeries)

Greater collaboration among physicians committed to global surgery

Better international relations between physicians in the local global community and colleagues in the U.S.

Improved coordination between surgical teams to maximize the value created for patients needing their expertise

Greater understanding of cultural differences leading to expanded expertise for both the visiting and hosting individuals

Continuous surgical education and training through Video and regularly scheduled clinical conferences

Greater access to international research efforts and solutions reported outside the U.S.

Opportunity to attract and retain medical students, residents and attending physicians with language proficiency and cultural expertise most appropriate for a particular global surgery

Establishment of more international partnerships for global surgery programs 
to teach basic and complex surgical techniques and provides a venue where surgical innovations can be shared within members of the same surgical specialty" [12]. Creating a global network will benefit all sites, encourage the development and sharing of patient resources and permit participants from dozens of nations to contribute to the collective expertise.

As with any major change, there will be new challenges. Some organizations and participants will have difficulty making the necessary cultural adjustments. Others won't find the experience as enjoyable as a onetime surgical trip. In contrast, those groups that want to foster a long-term relationship, and focus on cultural awareness and sensitivity, will have the highest probability of success. In the end, moving from a single trip focus to a model that emphasizes building a lasting relationship will prove most valuable for all involved.

Ask participants about a surgical trip abroad they volunteered for, and consistently, they recount how personally fulfilling it was for them. Most say they gained more from the experience than they contributed. Moving into "Generation 3" of the global surgical experience will prove to be not only "life-changing," but also "life-lasting". As medical students, residents and attending physicians learn from their colleagues across the globe, they will become better physicians, and be able to provide improved healthcare to the growing number of patients from diverse backgrounds in their own practices.

\section{Conclusions}

Global health missions have evolved over time from focusing on providing care and training American residents to educating and training local physicians. This has improved continuity of care, reduced cultural gaps, and decreased tensions with local doctors. In spite of these advances, the interactions between doctors from the host country and American physicians tend to be episodic, and without guaranteed stability for the future. We believe the next evolution of global health, Generation 3, will improve the global health experience for patients, physicians in these distant geographies and volunteer American doctors. This next generation of global surgery will focus on developing long-lasting personal relationships and use modern tools such as video to provide continuous learning between trips, reduce cultural barriers and encourage greater participation by medical students, residents and volunteer physicians who share the same language and background.

Moving from the currentgeneration to thenext willbe uncomfortable for some and pose significant cultural barriers that will need to be overcome. In spite of the steepness of the learning curve, we are confident that the result will be a more fulfilling experience for the participants and better outcomes for patients. In addition, we are hopeful that the closer integration will allow global health surgical programs to become more research and academically focused, and create the opportunity to develop training and educational materials for all. Finally, we believe that through global networks, more information can be shared, outcomes compared, and improvements and education facilitated. Advances in global surgery have paralleled changes in resident training and overall health care delivery in the U.S. As residency programs increasingly focus on cultural awareness and use new technology to meet the challenges of a more diverse domestic population, so, too, can global health programs apply some of these same approaches to improving the global health experience for both the sponsoring institutions and the patients receiving surgical care. Once this next evolution in global health occurs, few will want to return to the approaches of the past.

\section{References}

1. Shaye DA (2016) Global Cleft Surgery. Facial Plast Surg 32: 209-212. [Crossref]

2. Heymann DL, Liu J, Lillywhite L (2016) Partnerships, Not Parachutes, for Zika Research. N Engl J Med 374: 1506-1509. [Crossref]

3. Mackey TK, Liang BA (2012) Rebalancing brain drain: exploring resource reallocation to address health worker migration and promote global health. Health Policy 107: 6673. [Crossref]

4. Pearl RM (2014) Kaiser Permanente Northern California: Current experiences with internet, mobile, and video technologies. Health Affairs 33: 251-257. [Crossref]

5. Hunter DJ, Fineberg HV (2014) Convergence to common purpose in global health. $N$ Engl J Med 370: 1753-1755. [Crossref]

6. Mutabdzic D, Azzie G (2016) Uncovering the Hidden Curriculum of Global Health Electives. Ann Surg 263: 853-854. [Crossref]

7. Consortium of Universities for Global Health. Available at: http://www.cugh.org. Accessed April 24, 2016.

8. Hoyler M, Finlayson SR, McClain CD, Meara JG, Hagander L (2014) Shortage of doctors, shortage of data: a review of the global surgery, obstetrics, and anesthesia workforce literature. World J Surg 38: 269-280. [Crossref]

9. Nayar HS, Salyapongse AN, Mount DL, Bentz ML (2015) The Current State of Globa Surgery Training in Plastic Surgery Residency. Plast Reconstr Surg 136: 830e-7e. [Crossref]

10. Grossman L (2014) The man who wired the world: Mark Zuckerberg's crusade to put every single human being online. TIME 184: 30-40.

11. Davis MC, Can DD, Pindrik J, Rocque BG, Johnston JM (2016) Virtual interactive presence in global surgical education: international collaboration through augmented reality. Journal of Neurosurgery 86: 103-111. [Crossref]

12. CSurgeries (2016) Available at: http://www.csurgeries.com. Accessed April 24, 2016.

Copyright: (C2016 Chao J. This is an open-access article distributed under the terms of the Creative Commons Attribution License, which permits unrestricted use, distribution, and reproduction in any medium, provided the original author and source are credited. 